\title{
CANCER
}

\section{Distinct microenvironments in primary and transplant tumor models determine response to therapy}

Wisdom, A.J. et al. Nat. Commun. 11, 6410 (2020)

Strong preclinical evidence suggests that radiation therapy (RT) synergizes with immunotherapy to enhance antitumor immune responses and improve therapeutic efficacy in animal models of cancer. These observations have led to multiple clinical trials to evaluate the efficacy of the combination of radiotherapy with immunotherapy in patients with cancer, but to date the results of these trials have been disappointing.

Most preclinical studies use transplant tumor models, which do not fully recapitulate the complex nature of human cancers. Transplant models might overestimate patient response and explain clinical trial failures. New work published in Nature Communications supports this theory by showing that immunotherapy and RT can cure transplanted tumors in syngeneic mice but fail to cure mice bearing primary sarcomas from the same model system. The findings also suggest that primary and transplant tumor models have distinct immune landscapes that determine response to therapy.
In their study, investigators led by Amy J. Wisdom from Duke University Medical Center used a novel high-mutation model of murine sarcoma in which they induced primary tumors in the gastrocnemius muscle by deleting Trp53 in Trp53fl/fl mice with an adenovirus expressing Cre recombinase, followed by the injection of a carcinogen (MCA). Transplant tumors were initiated by injecting a cell line from a primary p53/MCA sarcoma into the gastrocnemius muscle of syngeneic mice. Mice from both models were treated with anti-PD-1 or isotype control antibody and 0 or $20 \mathrm{~Gy}$ of RT when tumors reached $>70 \mathrm{~mm}^{3}$. Survival analysis revealed that combining PD-1 blockade and 20 Gy RT cured mice with transplants, but failed to cure mice with primary p53/MCA sarcomas.

The team demonstrated that primary tumor resistance to immunotherapy was driven by the reshaping of the immune system during the development and progression of the tumor. Further analysis of the tumor environment revealed that transplanted tumors were enriched for activated $\mathrm{CD} 8^{+} \mathrm{T}$ cells and $\mathrm{PD}-\mathrm{L}^{+}$ macrophages, closely resembling highly inflamed human sarcomas that respond to PD-1 checkpoint blockade, whereas primary tumors, which coevolve with the immune system, recapitulated the immune-low microenvironment and resistance to $\mathrm{PD}-1$ blockade seen in most patient sarcomas.

"Our findings suggest that transplant tumor models may recapitulate the immune microenvironment of highly inflamed tumor subtypes that are likely to respond to immunotherapy, but do not resemble the majority of human cancers, which are resistant to immunotherapy. Performing complementary studies in transplant and autochthonous mouse models may thus increase the success of translation of immunotherapies from preclinical studies to patients," conclude the investigators in their report.

\section{Alexandra Le Bras}

Published online: 15 January 2021 https://doi.org/10.1038/s41684-021-00712-3

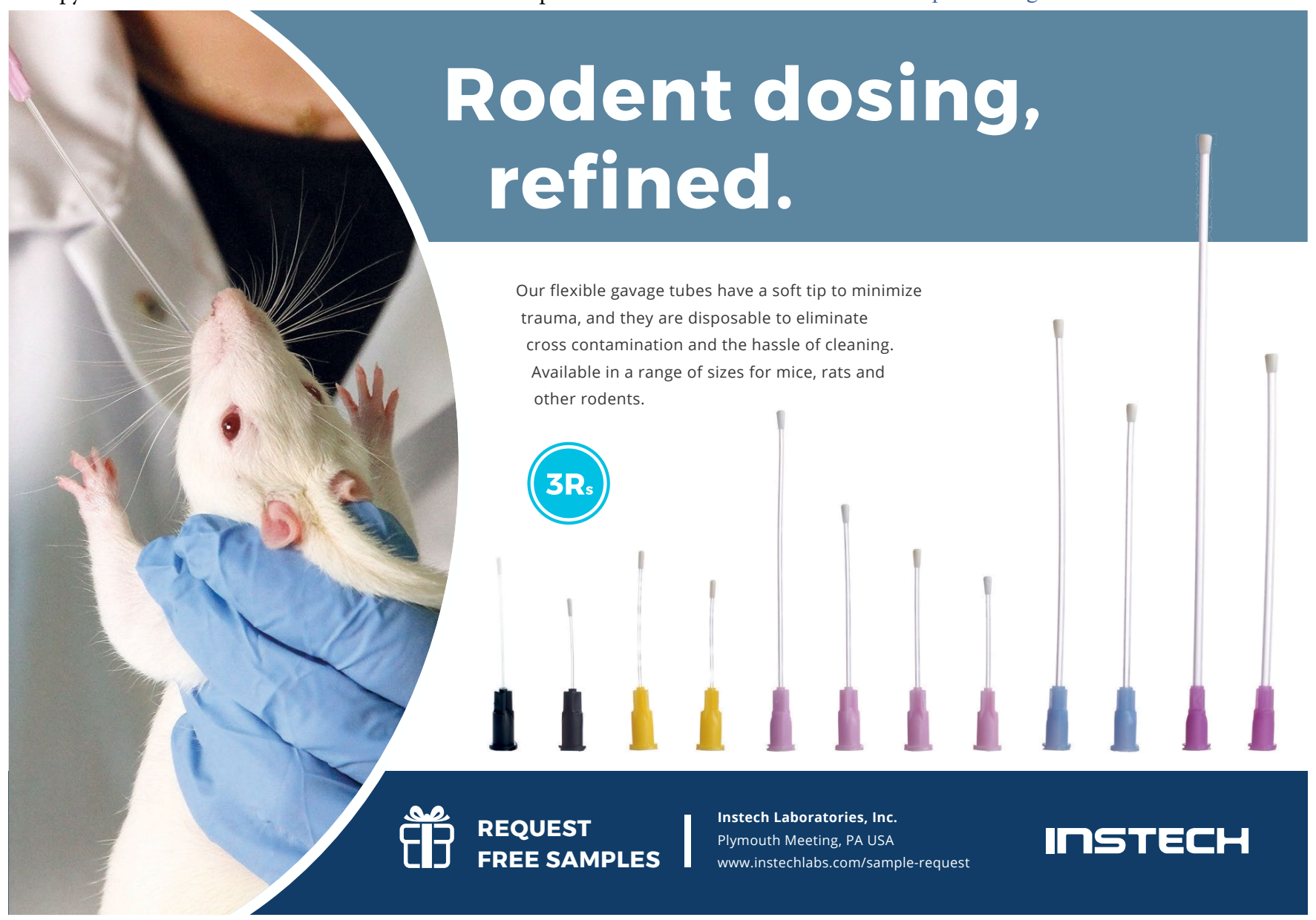

